EXTENDED REPORT

\title{
Transporter associated protein expression in uveal melanoma
}

\author{
S Krishnakumar, S A Lakshmi, D Abhyankar, J Biswas
}

Br J Ophthalmol 2004;88:925-928. doi: 10.1136/bjo.2003.018457

See end of article for authors' affiliations

.....................

Correspondence to: Dr S Krishnakumar, Vision Research Foundation, Sankara Nethralaya, 18 College Road, Chennai-600 006, Tamil Nadu, India; drkrishnakumar_2000@ yahoo.com

Accepted for publication 3 June 2003
Background/aim: Transporter associated protein (TAP) is important for presenting peptides to major histocompatibility complex (MHC) class I molecules. Defects in TAP lead to decreased MHC class I expression. The immunoexpression of human leucocyte antigen (HLA) class I molecules and the TAPI subunit were studied in primary uveal melanomas and correlated with the cell types and extrascleral extension.

Methods: The HLA class I antigen and TAP1 subunit were analysed by immunoperoxidase staining with monoclonal antibodies on 45 primary uveal melanoma archival specimens. The tumours were divided into two groups-group A: tumours with no extrascleral extension; group B tumours with extrascleral extension/liver metastasis. Immunoanalysis was done by a semiquantitative method.

Results: HLA class I antigen and TAP1 were decreased in 35 of 35 tumours with no extrascleral extension and positive in six of 10 tumours with liver metastasis. Decreased immunoexpression of HLA class I antigen and TAP1 in uveal melanomas with no extrascleral extension was significant $(p<0.001)$. HLA class I antigen and TAP1 were negative in spindle cell melanomas $(p<0.001)$.

Conclusions: HLA class I antigen and TAPI expression were decreased in uveal melanomas with no extrascleral extension and in spindle cell melanomas. Decreased expression of TAP1 may lead to decreased expression of HLA class I antigen in uveal melanomas. This preliminary observation deserves further investigation, which may shed more light on the immune escape mechanisms of this tumour and thus enable novel therapeutic strategies.
$\mathrm{T}$ he therapy of uveal melanoma remains problematic owing to the high rate of metastatic dissemination, irrespective of the success of treatment of the primary tumour. ${ }^{1}$ When metastatic disease is diagnosed, patient survival is usually less than 1 year. It is assumed that tumour dissemination occurs before primary tumour therapy. ${ }^{2}$ Immunotherapy has generated a lot of interest in the treatment of uveal melanoma.

Malignant transformation of cells is frequently associated with abnormalities in HLA. These abnormalities play a part in the clinical course of the disease, because HLA antigens mediate interactions of tumour cells with $\mathrm{T}$ cells and natural killer (NK) cells. ${ }^{3}$ Analysis of uveal melanoma lesions has shown associations between HLA class I antigen downregulation and favourable outcome, suggesting the susceptibility of ocular melanoma to NK cell mediated cytolysis. ${ }^{45}$

Antigen processing molecules are important for the expression of HLA class I antigens on the tumour surface. ${ }^{6}$ TAP (transporter associated protein) an important component in the antigen processing machinery, is a membrane spanning heterodimer consisting of two proteins: TAPI and TAP2. Both belong to the family of ATP binding cassette proteins found in the membranes of many cells; these proteins mediate ATP dependent transport of peptides. Peptides generated in the cytosol by the proteasomes are translocated by TAP into the rough endoplasmic reticulum by a process that requires ATP. The TAP1 and TAP2 genes map within the class II MHC region. ${ }^{7}$ Deficiency of either TAPI or TAP2 leads to decreased MHC class I expression.

Abnormalities of the MHC class I expression have frequently been associated with impaired TAP expression in human tumours. ${ }^{89}$ The purpose of this study was to investigate the immunoexpression of HLA class I antigen and TAPl subunit on formalin fixed, paraffin embedded primary uveal melanoma lesions with monoclonal antibodies and correlate this expression with the cell types and extrascleral extension.

\section{MATERIALS AND METHODS \\ Tumour material}

We had earlier published a large series on uveal melanomas in Asian Indians. ${ }^{10}$ Patients who underwent enucleation for uveal melanoma at our institute between 1994 and 2000 were included in the study. Iris melanomas, metastatic melanoma of the uvea, melanomas with extensive necrosis, and orbital cellulitis were excluded. There were 45 cases with sufficient tumour tissue for analysis. Patients consisted of $30 \mathrm{men}$ and 15 women, with a mean age of 45 years. The right eye was involved in 29 cases and the left eye in 16.

The study was reviewed and approved by the local ethics committee of our institute, and the committee deemed that it conformed to the generally accepted principles of research, in accordance with the Helsinki Declaration.

The tumours were formalin fixed for 24 hours at room temperature and then paraffin embedded for histological examination. The specimens were stained with haematoxylin and eosin, and histological evaluation was performed with a light microscope. Cell type was classified by using the modified Callender's classification ${ }^{11}$ : spindle type, mixed type, or epithelioid type. The presence of extrascleral extension was noted. Clinical details were obtained from the files of the patients. The tumours were divided in to two groups-group A: tumours with no extrascleral extension; and group B: tumours with extrascleral invasion/liver metastasis. The minimum follow up for group A tumours was 20 months and the maximum follow up was for

Abbreviations: HLA, human leucocyte antigen; MHC, major histocompatibility complex; TAP, transporter associated protein 
60 months. The minimum follow up for group B tumours was 18 months and the maximum follow up was 60 months.

\section{Monoclonal antibodies}

The affinity purified locus specific mouse anti-human monoclonal antibody (mAb) HC-10 that recognises all HLA-B heavy chains and anti-human monoclonal antibody (mAb) anti-TAPl mAb T0-1 were used. The antibodies are target specific and exhibits no cross reactivity. ${ }^{12}$ Labelled streptavidin biotin kit was purchased from Dako Laboratories (Denmark).

\section{Immunohistochemical staining protocol}

The $4 \mu \mathrm{m}$ paraffin embedded sections were mounted on silane coated glass slides. Immunostaining of tissue sections was performed using labelled streptavidin by indirect immunoperoxidase technique. Tissue sections were then deparaffinised, rehydrated, and bleached before the immunohistochemical procedure and endogenous peroxidase was blocked with hydrogen peroxide for 10 minutes at room temperature. No antigen retrieval was performed before antibody incubation.

Tissue sections were then rinsed in TRIS buffered saline (TBS) at pH 7.6 and incubated with primary antibody for 1 hour. This was followed by a sequential 40 minute incubation with biotinylated secondary antibody and streptavidin labelled to horseradish peroxidase (Dako). Sections were washed with TBS between incubation. The peroxidase reaction was developed for 5 minutes using commercially available 3,3' diaminobenzidine and counterstained with haematoxylin. The staining of adjacent normal structures (that is, lymphoid and macrophage cells) was used as an internal control to evaluate the staining intensity of malignant cells. For negative control the primary antibody was omitted and non-immune serum was used in the immunostaining.

\section{Assessment of immunohistochemical results}

A pathologist without knowledge of the clinical history performed all scoring and interpretation of immunohistochemical results. Cells were considered positive when the cytoplasm was stained. The staining was assessed under $40 \times$ high power objective and by studying 10 fields. The tumours were scored by assessing the proportion of stained cells and the intensity of the stained tumour cells.

The staining intensity was scored as - (absent), +- (dull), and + (bright).) The tumours were graded as follows: positive (greater than $75 \%$ cells stained and with bright intensity of staining) (75\%-95\% of cells stained, but with the majority of cells exhibiting a dull intensity of staining), heterogeneous $(25 \%-75 \%$ of the cells stained, mainly at a dull intensity) with the percentage of cells expressed to nearest 10\%), and negative (less than $25 \%$ of cells stained at a minimum of low intensity) (HLA expression in cancer, International Histocompatibility Working Group, Project description, www.ihwg.org).

\section{Statistical analysis}

For statistical analysis the negative and heterogeneous HLA class I antigen and TAPl expression were considered as decreased immunoexpression. Decreased HLA class I antigen and TAPl immunoexpression were compared with the positive HLA class I antigen and TAPl expression in the two groups of tumours. Similarly, immunoreactivity was compared between the spindle cell melanoma and the nonspindle melanomas comprising both the mixed and epithelioid cell types using Fisher's exact test.

\section{RESULTS \\ Histopathology}

The clinical and immunohistochemical data of the two groups of tumours are given in tables 1 and 2 respectively. Among the 45 uveal melanomas, 35 were from the choroid, nine were from both ciliary body and choroid, and one was a diffuse uveal melanoma. ${ }^{13}$ Histological cell identification showed 17 spindle cell melanomas, 16 mixed cell, and 12 epithelioid cell melanomas. Thirty five tumours (group A) had no extrascleral extension. Extrascleral extension was seen in 10 tumours (group B), of which six had liver metastasis.

\section{HLA class I antigen and TAP 1 immunoreactivity and extrascleral extension}

Immunohistochemical staining revealed cytoplasmic localisation of HLA class I antigen and TAP1. No positive staining was obtained in any of the negative controls. Table 1 shows the HLA class I antigen and TAP 1 immunoreactivity with regard to extension in uveal melanomas. Among the 35 melanomas with no extrascleral extension (group A) HLA class I antigen and TAPI immunoexpression were decreased in all 35 of 35 tumours (negative with absent staining in 30 of $35(88 \%)$ and heterogeneous with $30 \%$ to $40 \%$ cells stained with dull staining in five of 35 (12\%) tumours). Among the 10 tumours with extrascleral extension (group B) HLA class I antigen and TAP 1 immunoexpression were positive in six of 10 tumours, (bright staining of $>70 \%$ of cells in four tumours and $90 \%$ of cells stained with dull intensity in two tumours) with liver metastasis and decreased in four of 10 tumours (heterogeneous with $40 \%$ cells stained with dull intensity in three tumours and negative with absent staining in one tumour) with only extrascleral extension and no liver metastasis up to the recent follow up. Decreased HLA class I antigen and TAPl immunoreactivity were significant in tumours (group A) with no extrascleral extension $(p<0.001)$.

\section{HLA class I antigen and TAP I immunoreactivity and cell types}

Table 2 shows the HLA class I antigen and TAPI immunoreactivity with regard to cell types. Among the 17 spindle melanomas, HLA class I antigen and TAPl were negative (absent staining) in 16 of 17 (94\%) tumours, heterogeneous (40\% cells stained with dull intensity) in one of 17 (6\%) tumours. Among the 28 non-spindle cell tumours, HLA class I antigen and TAP were negative (absent staining) in 15 of 28 $(54 \%)$ tumours, heterogeneous in seven of $28(25 \%)$ tumours

\begin{tabular}{|c|c|c|c|}
\hline \multirow[b]{2}{*}{ Group } & \multicolumn{3}{|c|}{ HLA class I antigen and TAPI immunoreactivity } \\
\hline & Negative & Heterogeneous & Positive \\
\hline $\begin{array}{l}A(n=35) \\
B(n=10)\end{array}$ & $\begin{array}{l}30 \\
1\end{array}$ & $\begin{array}{l}5 \\
3\end{array}$ & $\overline{6}$ \\
\hline
\end{tabular}


Table 2 TAP1 immunoreactivity and correlation of cell types

\begin{tabular}{llll}
\hline \multirow{2}{*}{ Cell types } & \multicolumn{2}{l}{ HLA class I antigen and TAP 1 immunoreactivity } \\
\cline { 2 - 4 } \cline { 2 - 4 } & Negative & Heterogeneous & Positive \\
\hline Spindle cell melanoma & 16 & 1 & - \\
Mixed cell melanoma & 11 & 2 & 3 \\
Epithelioid cell melanoma & 4 & 5 & 3 \\
\hline
\end{tabular}
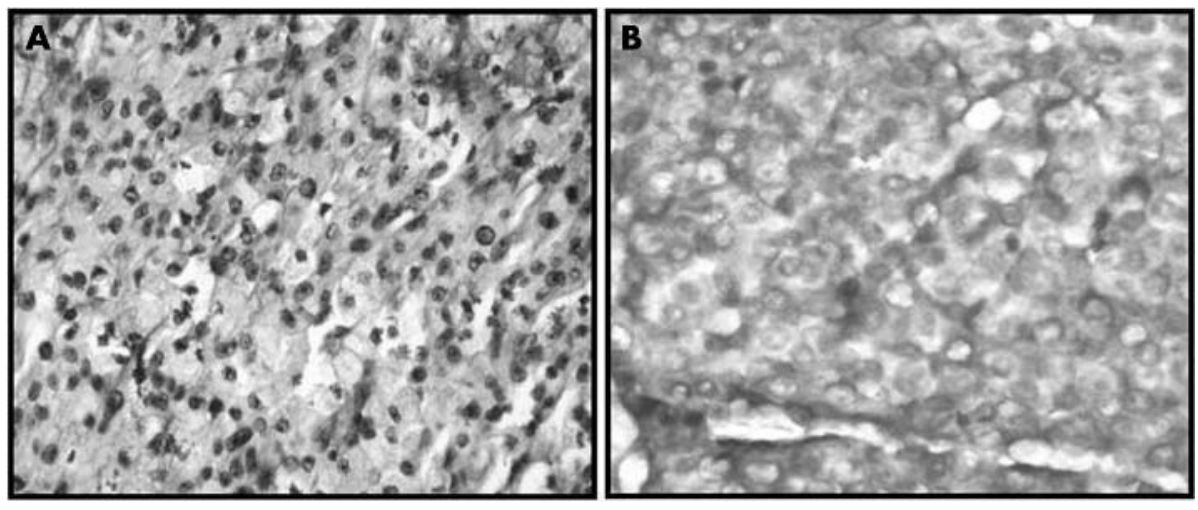

Figure 1 Immunohistochemistry of TAP1. (A) The negative immunoreactivity of TAPI in the spindle cell uveal melanoma. (B) The positive immunoreactivity of TAP1 in the choroidal melanoma with hepatic metastasis (magnification $\times 40$ ).

(40-50\% cells stained with dull intensity), and positive in six of $28(21 \%)$ tumours (bright staining in $70 \%$ of cells in four tumours and dull staining in $90 \%$ of cells in two tumours). Negative HLA class I antigen and TAP 1 immunoexpression were significant in the spindle cell melanomas $(\mathrm{p}<0.001)$. Figure IA shows negative TAPl in spindle cell uveal melanoma and figure $1 \mathrm{~B}$ shows positive TAPl expression in choroidal melanoma with hepatic metastasis.

\section{DISCUSSION}

HLA class I antigen and TAPl expressions were decreased in uveal melanomas with no extrascleral extension $(\mathrm{p}<0.001)$ and positive in tumours with liver metastasis. Decreased HLA class I antigen and TAPI immunoreactivity were significant in the spindle cell melanomas $(p<0.001)$ Our results of HLA class I antigen expression in uveal melanoma are similar to the studies of HLA class I antigen expression in uveal melanomas where decreased HLA antigens correlated with a favourable outcome and decreased immunoexpression was significant in the spindle cell choroidal melanomas. ${ }^{45}$

However, our results on TAPl expression are discordant with a previous study, ${ }^{14}$ in which reduced expression of TAPl in uveal melanoma correlated with metastasis. However, Jager $e t a l^{15}$ observed that ocular melanoma cell lines express poor, if any, levels of both peptide transporter molecules TAP1/TAP2, as well as tapasin, as measured by western blot analysis, and proteasomes were also undetectable in ocular melanoma cells propagated in culture as long term cell lines.

The molecular basis of the lack of HLA expression on uveal melanoma has not yet been elucidated. ${ }^{15}$ Locus specific downregulation of HLA antigens can be transcriptionally mediated or caused by genetic defects. ${ }^{16}{ }^{17}$ Immunohistochemical methods cannot discriminate between these two possibilities. The frequency of locus specific downregulation in uveal melanoma is very high, $33 \%$ and $40 \%$ for HLA-A and B respectively. ${ }^{15}$ In our study HLA class I antigen was downregulated in 39 tumours $(88 \%)$; this is similar to results of a study by Erricson et al. ${ }^{5}$

In this regard, the concordant downregulation of HLA class I antigen and TAP units in a high percentage of uveal melanomas in our study suggests defects in the regulatory mechanisms that control their expression and not structural defects in the corresponding genes. It is unlikely that mutations are present in multiple genes encoding HLA class I antigen and TAPI in uveal melanoma.

Recently, Jager et al ${ }^{15}$ investigated the effects of cytokines on the MHC class I pathway in ocular melanoma cell lines. They found that interferon (IFN) treatment resulted in significant upregulation of TAP1/TAP2 proteins, immunoproteasomal subunits, and the MHC class I heavy chain in ocular melanoma cell lines. These changes were paralleled by the upregulation of MHC class I at the cell surface along with the increase in average half life of the complexes. This indicated transcriptional defects and that ocular melanomas have functional HLA class I presentation machinery capable of responding to IFN type 2 .

Thus, antigen processing and presentation by class I MHC molecules generally require assembly with peptide epitopes generated by the proteasome and transported into the endoplasmic reticulum by the TAP. Defects in TAP could lead to decreased peptide availability and decreased HLA class I antigen expression. However, recently, TAP independent pathways supporting class I MHC mediated presentation of exogenous antigens, as well as of endogenously synthesised antigens, has been described. This pathway is proteasome independent. Cell surface expression of these TAP independent class I complexes is modulated by tapasin levels and enhanced by IFN gamma. ${ }^{18}$

In conclusion, our preliminary data suggest that majority of the primary uveal melanomas with no extrascleral extension or liver metastasis have a decreased HLA class I antigen and TAPl expression and tumours with liver metastasis, have a strong HLA class I antigen and TAPI expression. Biologically, our findings suggest a potential implication of TAPl in tumour progression. Further studies should clarify whether the primary uveal melanomas with no liver metastasis express strong TAP $1^{14}$ or very low TAP 1 from our data, supported by Jager et al study on ocular melanoma cell lines. ${ }^{15}$ This has great implications in the immunotherapy of uveal melanomas. 


\section{ACKNOWLEDGEMENTS}

This work was possible because of grants from Vision Research Foundation, Sankara Nethralaya, Chennai, India. The monoclonal antibody was a gift from Dr Soldano Ferrone, Department of Immunology, Roswell Park Cancer Institute, Buffalo, New York USA. We thank Ms Vaijayanthi P for her excellent technical work and Mr Sukumar for the statistical assistance.

\section{Authors' affiliations}

S Krishnakumar, S A Lakshmi, J Biswas, Department of Ocular Pathology, Vision Research Foundation, Sankara Nethralaya, Chennai, India

D Abhyankar, Evanston North Western Health Care, Evanston, IL, USA

Commercial relationships: Nil.

\section{REFERENCES}

1 Raipal S, Moore R, Karakousis CP. Survival in metastatic ocular melanoma. Cancer 1983;52:334-6.

2 Kath R, Hayungs J, Bornfeld N, et al. Prognosis and treatment of disseminated uveal melanoma. Cancer 1993;72:2219-23.

3 Garrido F, Cabrera T, Concha A, et al. Natural history of HLA expression during tumor development. Immunol Today 1993;14:49-99.

4 Blom DJR, Luyten GPM, Mooy CM, et al. Human leukocyte antigen class I expression: marker of poor prognosis in uveal melanoma. Invest Ophthalmol Vis Sci 1997;38:1865-72.

5 Erricson C, Seregard S, Bartolazzi A, et al. Association of HLA class I and Class II antigen expression and mortality in uveal melanoma. Invest Ophthalmol Vis Sci 2001;42:2153-6.
6 Pamer E, Cresswell P. Mechanisms of MHC class I restricted antigen processing. Annu Rev Immunol 1998;16:323-58.

7 Momburg F, Hammerling GJ. Generation and TAP mediated transport of peptides for MHC class I molecules. Adv Immunol 1998;68:191-256.

8 Seliger B, Maeurer MJ, Ferrone S. TAP off-tumors on. Immunol Today 1997; 18:292-9.

9 Johnsen AK, Templeton DJ, Sy M, et al. Deficiency of transporter for antigen presentation (TAP) in tumor cells allows evasion of immune surveillance and increases tumorigenesis. J Immunol 1999;163:4224-31.

10 Biswas J, Krishnakumar S, Shanmugam MP. Uveal melanoma in Asian Indians: a clinicopathological study. Arch Ophthalmol 2002; 120:522-3.

11 Callender GR. Malignant melanotic tumors of the eye: a study of histologic type in 111 cases. Trans Am Acad Ophthamol Otolaryngol 1931;36:131-42.

12 Kishore R, Hicklin DJ, Dellaratta DV, et al. Development and characterization of mouse anti-human LMP2, LMP7, TAP1 and TAP2 monoclonal antibodies. Tissue Antigens 1998;51:129-40.

13 Biswas J, Raghavendran R, Ratra V, et al. Diffuse malignant melanoma of the choroid simulating metastatic tumor to the choroid. Ind J Ophthalmol 2000;48:137-40.

14 Cresswell AC, Sisley K, Lawe D, et al. Reduced expression of TAP- 1 and TAP2 in posterior uveal melanoma is associated with progression to metastatic disease. Melanoma Res 2002;11:275-81

15 Jager MJ, Hurks HM, Levitskaya J, et al. HLA expression in uveal melanoma: there is no rule without some exception. Hum Immunol 2002:63:444-51.

16 Hicklin DJ, Marincola FM, Ferrone S. HLA class I antigen down regulation in human cancers. T cell immunotherapy revives an old story. Mod Med Today 1999;5:178-88.

17 Garrido F, Ruiz-cabello F, Cabrera T, et al. Implications for immunosurveillance of altered HLA class I phenotypes in human tumours. Immunol Today 1997;18:89-95.

18 Fromm SV, Duady-Ben Yaakov S, Schechter C, et al. Assembly and cell surface expression of TAP-independent, chloroquine-sensitive and interferongamma-inducible class I MHC complexes in transformed fibroblast cell lines are regulated by tapasin. Cell Immunol 2002;215:207-18. 